\title{
Síndrome de Lemierre: Una entidad clínica casi en el olvido. Reporte de tres casos y revisión de la literatura
}

\author{
Dres. Ignacio Maldonado Sch ${ }^{(1)}$, José M Gutiérrez $C^{(1)}$, Anita Wilkens $R^{(2)}$, Carolina Weitz $R^{(3)}$, Alberto Rojas $A^{(2)}$, \\ Cristian Varela $U^{(1)}$.
}

1. Radiólogo Unidad de Radiología Cuerpo, Departamento de Imagenología, Clínica Dávila, docente Universidad de los Andes y Universidad Mayor. Santiago - Chile.

2. Médico-Cirujano, pasantía de capacitación, Departamento de Imagenología, Clínica Dávila. Santiago - Chile.

3. Residente de Radiología, Universidad de Los Andes. Santiago - Chile.

\section{Lemierre's syndrome: An almost forgotten clinical entity. Three case reports and Literature review}

\begin{abstract}
Lemierre's syndrome is a rare disease that affects young adults and is mainly caused by Fusobacterium necrophorum and occasionally by other anaerobic bacteria of the species. This syndrome is characterized by a throat infection complicated with septic thrombophlebitis of the internal jugular vein and septic emboli mainly to the lungs. In the pre-antibiotic era its evolution was often fatal. Since the 1960's this syndrome has rarely been reported given the extensive use of penicillin en pharyngeal infections. Currently the incidence of Lemierre's syndrome is about one in a million. Currently, since imaging has a key role in the early diagnosis of this syndrome, the radiologist should be aware of and recognize its manifestations. Three cases of Lemierre's syndrome and a literature review are presented.

Keywords (MeSH-Pubmed): Diagnostic Imaging (D003952), Lemierre Syndrome. (D057831), Pulmonary embolism (D011655).
\end{abstract}

Resumen: El síndrome de Lemierre es una enfermedad rara que afecta a adultos jóvenes y es causada principalmente por Fusobacterium necrophorum y ocasionalmente por otras bacterias anaerobias de la especie. Este síndrome se caracteriza por una infección faríngea complicada con tromboflebitis séptica de la vena yugular interna y embolias sépticas principalmente a los pulmones. En la era pre-antibióticos su evolución era frecuentemente de curso fatal. Desde los años 60 este síndrome ha sido rara vez reportado dado el extensivo uso de penicilina en infecciones faríngeas. Actualmente la incidencia del síndrome de Lemierre es de aproximadamente uno en un millón. Dado que hoy en día las imágenes tienen un rol fundamental en el diagnóstico temprano de este síndrome el radiólogo debe estar al tanto y reconocer sus manifestaciones. Se presentan tres casos de Síndrome de Lemierre y una revisión de la literaratura. Palabras Clave (MESH-Pubmed): Diagnóstico por Imagen (D003952), Embolia Pulmonar (D011655), Síndrome de Lemierre (D057831).

Maldonado I, Gutiérrez JM, Wilkens A, Weitz C, Rojas A, Varela C. Síndrome de Lemierre: Una entidad clínica casi en el olvido. Reporte de tres casos y revisión de la literatura. Rev Chil Radiol 2015; 21(1): 34-40.

Correspondencia: Dr. Ignacio Maldonado Schoijet / imschoijet@gmail.com

Trabajo recibido el 26 de agosto de 2014. Aceptado para publicación el 25 de septiembre de 2014.

\section{Introducción}

El síndrome de Lemierre es una patología poco frecuente y potencialmente letal, que se origina como complicación de una infección orofaríngea que se extiende al espacio carotideo. Se asocia a tromboflebitis séptica de la vena yugular interna y embolias sépticas a distancia siendo el compromiso pulmonar el más frecuente ${ }^{(1)}$ (Figura 1).
Aunque inicialmente fue descrito en 1900 por Courmont y Cade, fue el microbiólogo francés André Lemierre (Figura 2) quien mejor caracterizó esta patología en 1936. Lemierre describió 20 casos de "septicemias anaeróbicas postangina", de los cuales 18 fallecieron. Este grupo de pacientes se presentaron inicialmente con un absceso faringoamigdaliano o periamigdaliano seguido de sensibilidad del músculo 
esternocleidomastoídeo, con el subsecuente desarrollo de embolias sépticas usualmente a pulmón y articulaciones. Los pacientes generalmente fallecían dentro de 7 a 15 días de iniciado el cuadro. Lemierre postuló que este síndrome era tan característico, que permitía realizar el diagnóstico incluso antes de los exámenes bacteriológicos ${ }^{(1,2)}$.

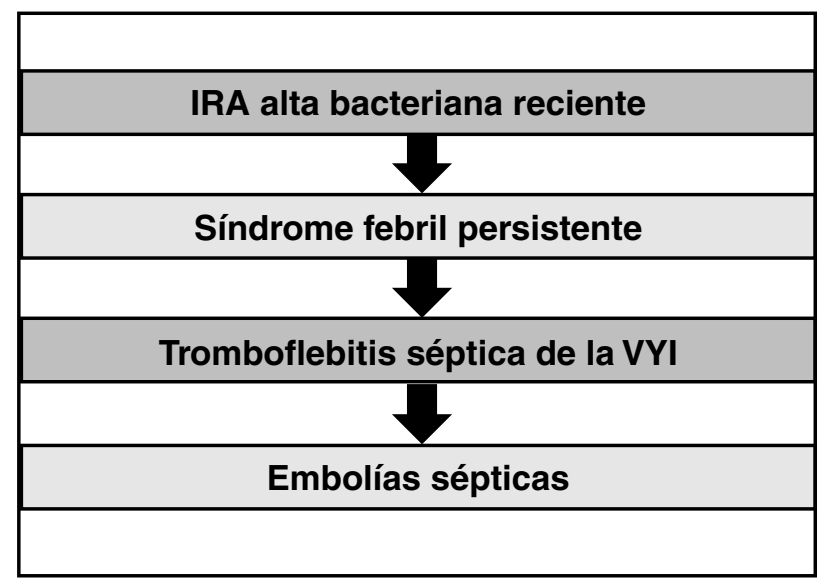

Figura 1. Secuencia de eventos en el sindrome de Lemierre.

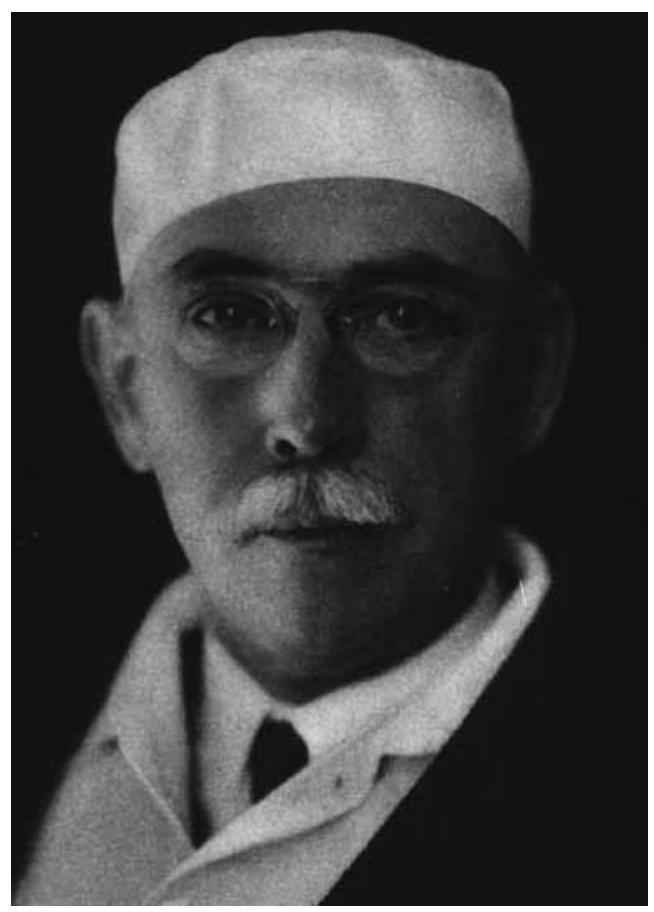

Figura 2. André Lemierre, bacteriólogo francés. En 1936 describe 20 casos de "septicemia anaeróbica post-angina", de los cuales 18 murieron. El síndrome se caracterizaba por infección orofaríngea que luego evoluciona a tromboflebitis de la vena yugular interna y posteriormente a embolías sépticas a distancia. Imagen: Lutétia, "Monsieur Le Professeur Lemierre photo", extraída de "Images from the History of Medicine (NLM)".
El patógeno más frecuente de este síndrome es el Fusobacterium necrophorum, una bacteria Gram negativa anaerobia estricta que forma parte de la flora normal de la orofaringe, tracto gastrointestinal y tracto genital femenino. A pesar de que este microorganismo está presente en $81 \%$ de los casos de síndrome Lemierre, una amplia variedad de otros patógenos aeróbicos y anaeróbicos han sido implicados incluyendo Staphilococo, Streptococo, Proteus, Bacteroides y Peptostreptococo. Ocasionalmente pueden encontrarse en forma aislada, pero es posible que esos casos reflejen la dificultad para cultivar organismos anaerobios estrictos ${ }^{(3,4)}$.

\section{Cuadro clínico}

El síndrome de Lemierre afecta típicamente a pacientes adolescentes y adultos jóvenes inmunocompetentes: más del $70 \%$ de los casos ocurren entre los 16 y 25 años. Se presenta generalmente como un cuadro séptico caracterizado por fiebre y odinofagia de varios días de evolución tras el antecedente de una faringitis aparentemente resuelta, siendo en el $87 \%$ de los casos las tonsilas palatinas y tejido peritonsilar el sitio de infección primaria. Otros focos infecciosos iniciales pueden corresponder a otitis media aguda, infecciones de origen dental y mastoiditis ${ }^{(1,3)}$.

En una segunda etapa existe invasión del espacio carotideo ocasionando tromboflebitis séptica de la vena yugular interna. Alrededor del $48 \%$ de los pacientes no presentan síntomas cervicales, sin embargo, pueden referir dolor y aumento de volumen cervical ${ }^{(5)}$. En las etapas finales existen siembras tromboembólicas sépticas que alcanzan órganos distantes, siendo el pulmón el sitio más común de diseminación $(80 \%)^{(6)}$ reportándose además complicaciones tales como: abscesos pulmonares, neumatoceles, derrame pleural, empiema y neumotórax ${ }^{(1,4)}$.

La artritis séptica era parte de la triada diagnóstica original del síndrome de Lemierre, sin embargo, actualmente es una presentación poco frecuente $(13-27 \%)^{(5)}$. Otras complicaciones poco comunes incluyen: osteomielitis, abscesos esplénicos y hepáticos, compromiso cutáneo y trombosis del seno cavernoso ${ }^{(3,4)}$.

Se ha propuesto establecer criterios diagnósticos estrictos de esta patología, dado que algunos autores incluyen dentro del síndrome de Lemierre la presencia de trombosis de vena yugular interna (por ejemplo, como complicación derivada del uso de vías venosas centrales) aún en ausencia de embolias sépticas a pulmón u otros órganos ${ }^{(1)}$ (Figura 3 ).

El tratamiento del síndrome de Lemierre consiste en terapia antibiótica prolongada con adecuada cobertura para anaerobios, especialmente dirigidos contra la cepa Fusobacterium. El esquema generalmente incluye penicilinas y metronidazol o monoterapia con clindamicina durante un período mínimo de 6 semanas. En cuanto al uso de anticoagulación sistémica 
se considera su uso cuando se acompaña de infarto cerebral o trombosis del seno cavernoso. Sin embargo, no existe evidencia que respalde su uso de forma generalizada en estos pacientes ${ }^{(3,4,5)}$.
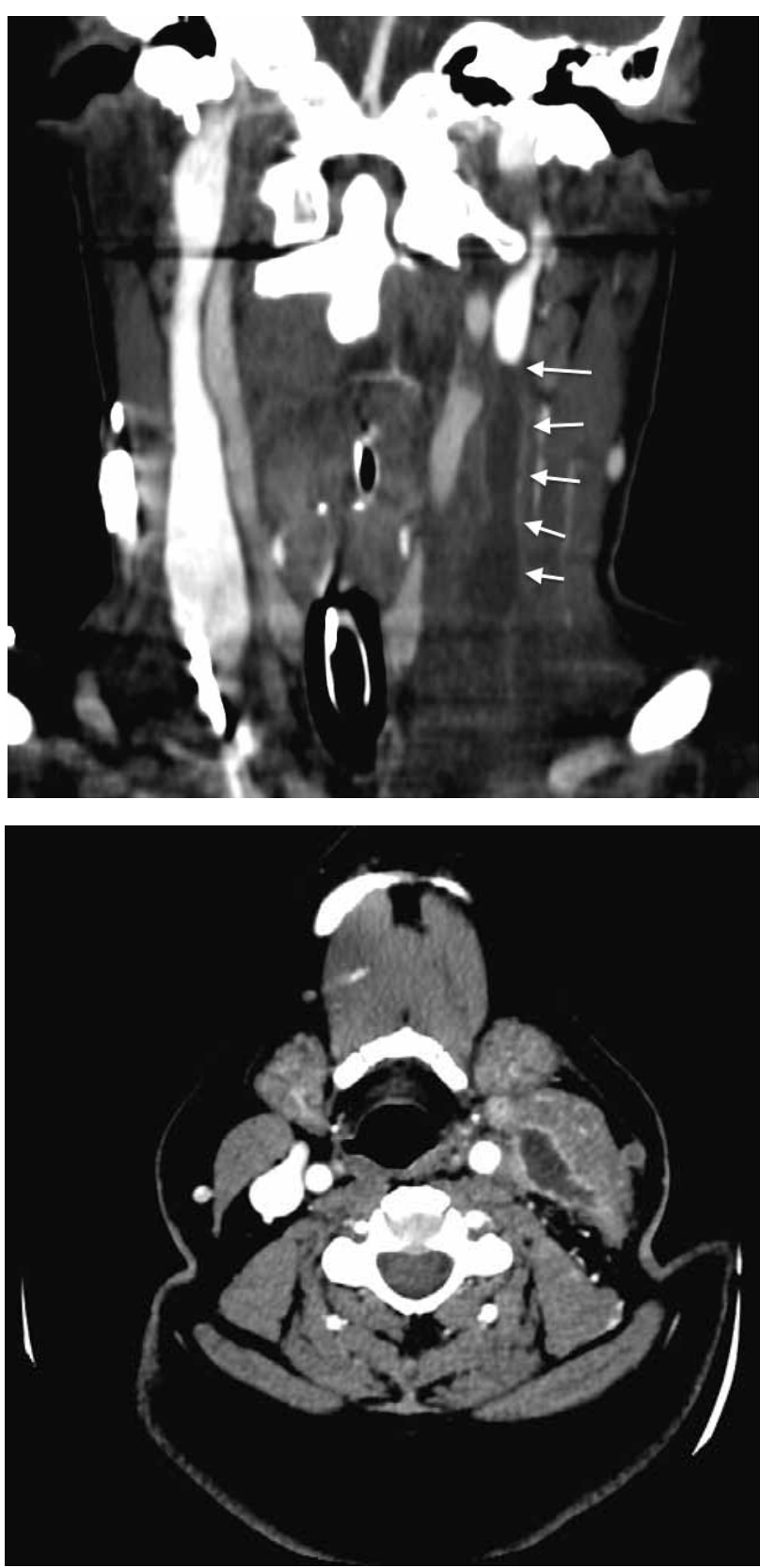

Figura 3. Caso 1, a) MPR coronal de TC cuello: Se observa la vena yugular izquierda expandida y ocupada por material hipodenso compatible con material trombótico b) corte axial TC de cuello: Se observa absceso en el compartimento vascular cervical izquierdo.

\section{Presentación de casos}

Se presenta una serie de tres casos con diagnóstico imagenológico de este síndrome, el primero trata de un paciente de sexo femenino y 57 años de edad, con antecedentes de hipertensión arterial.
Refiere cuadro de cervicalgia asociado a aumento de volumen cervical izquierdo, sin fiebre, tratado de forma ambulatoria con antiinflamatorios durante 10 días. Consulta en Servicio de Urgencias por persistencia de síntomas, por lo cual se solicita tomografía computada de cuello con contraste que evidencia absceso en el compartimento vascular cervical izquierdo subyacente al músculo esternocleidomastoideo, asociado a trombosis de vena yugular externa e interna izquierda. Estudio con AngioTC de tórax realizado posteriormente no demostró hallazgos patológicos, en especial, signos de embolias sépticas.

Como segundo caso, un paciente de sexo masculino, 16 años, sin antecedentes mórbidos. Consulta en Servicio de Urgencia por cuadro de 12 días de evolución caracterizado por fiebre, trismus, odinofagia y CEG tratado previamente con múltiples esquemas antibióticos. Al examen físico destaca febril $\left(40^{\circ} \mathrm{C}\right)$, faringe con exudado purulento, aumento de volumen amigdalino bilateral y adenopatías cervicales dolorosas. La radiografía de tórax de ingreso demuestra lesión radiolúcida cavitada, por lo cual se solicita TC de tórax que confirma la presencia de nódulos cavitados. Paciente evoluciona con aumento de volumen sensible en región lateral cervical derecha; se solicita Eco Doppler y TC de cuello con contraste, los cuales evidencian defecto de llenado en vena yugular interna derecha compatible con trombosis venosa.

Por último, tenemos un paciente de sexo femenino, 19 años, sin antecedentes mórbidos. Ingresa a Servicio de Urgencia febril, con cervicalgia y masa palpable en región cervical izquierda. TC de cuello y TC de tórax con contraste evidencian trombosis de vena yugular interna (VYI) izquierda y nódulos pulmonares, respectivamente. Evoluciona favorablemente luego de tratamiento antibiótico.

\section{Diagnóstico por imágenes}

El estudio con imágenes cumple un rol esencial en la confirmación diagnóstica cuando existe una adecuada sospecha clínica.

En la evaluación de las embolias sépticas es importante mencionar que los hallazgos radiológicos, particularmente en la tomografía computada (TC) de tórax, pueden estar presentes incluso antes que los hemocultivos estén positivos ${ }^{(7)}$. Aunque la $R x$ tórax no tiene igual sensibilidad que la TC, también puede mostrar hallazgos que permitan sospechar una embolia séptica. Entre los ellos se describen: pequeñas opacidades parenquimatosas generalmente bilaterales de distribución periférica y hacia las bases, de bordes mal definidos y variables en cuanto a su tamaño, luciendo como una neumopatía aguda habitual (área de condensación previa a la cavitación). Cuando estas lesiones se cavitan, tienden a observarse como lesiones cistoideas de pared fina ${ }^{(7-9)}$ (Figura 4). 
En el estudio por tomografía computada multicorte (TCMC) de tórax, las embolias sépticas presentan un espectro imagenológico que incluye áreas de opacidades en vidrio esmerilado, pequeñas opacidadas nodulares $(0,5-3 \mathrm{~cm}$ de diámetro) y focos de condensación con broncograma aéreo de localización subpleurales, los que tienden a presentar morfología en cuña (base pleural) simulando pequeños infartos pulmonares. Estas lesiones pueden exhibir grados variables de excavación dependiendo del estadío de evolución de la lesión, siguiendo una distribución de predominio periférica y hacia las bases pulmonares ${ }^{(9,10,11)}$ (Figura 5, Figura 11).
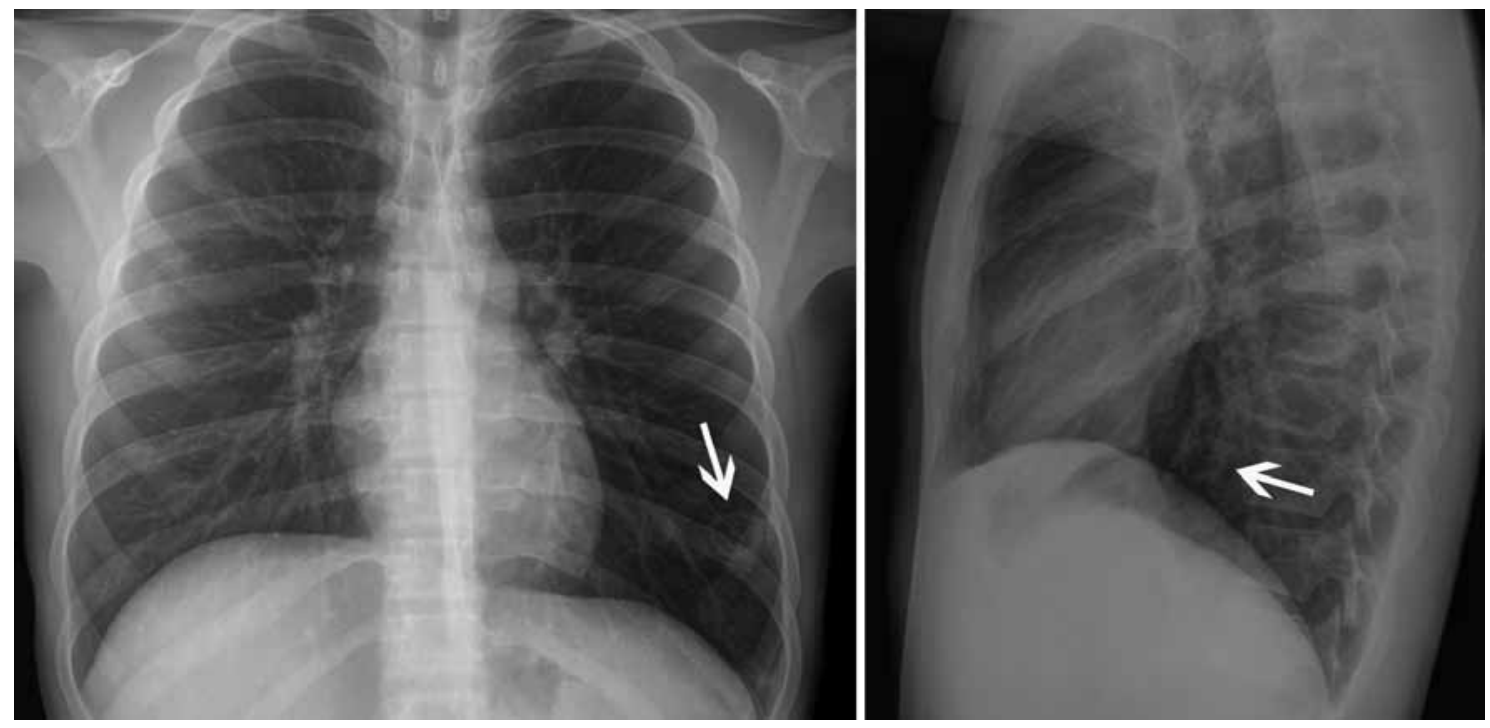

Figura 4. Caso 2. Rx tórax de ingreso: Evidencia una imagen radiolúciuda de aspecto cistoídea, de paredes relativamente finas de aprox $2 \mathrm{~cm}$ en el lóbulo inferior izquierdo, sospechosa de lesión cavitada.

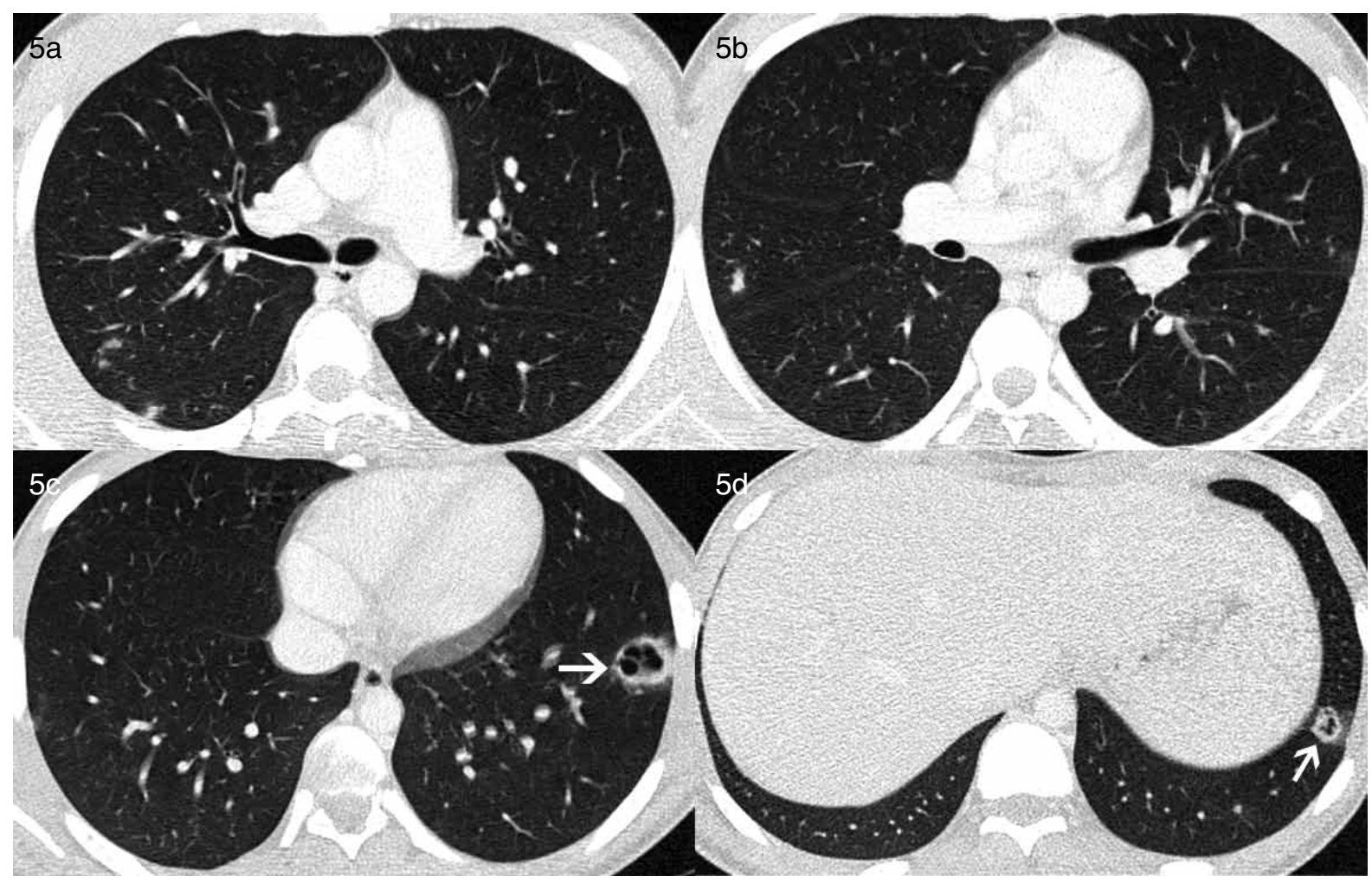

Figura 5. Caso 2, TC Tórax ventana pulmonar: El estudio confirmó la presencia de múltiples pequeñas opacidades nodulares subpleurales no mayores de $10 \mathrm{~mm}$ en el segmento postero basal del LSD y LM (flechas a, b), siendo visibles pequeños nódulos cavitados en la base del Lóbulo inferior izquierdo, uno de ellos, se corresponde con lo observado en $R x$ tórax previa (flechas $c, d)$. 
En ocasiones, se puede observar un pequeño vaso pulmonar que se orienta al espesor de la lesión, corresponde al "signo del vaso nutricio", que si bien es sensible, carece de especificidad puesto que ha sido reportado también en las metástasis pulmonares $(18-58 \%)^{(12)}$.

En la evaluación de los nódulos cavitados hay que considerar dentro del diagnóstico diferencial además de embolias sépticas: metástasis pulmonares, infecciones crónicas granulamatosas (hongos y TBC), linfoma (especialmente en pacientes con $\mathrm{VIH}$ ), nódulos reumatoídeos y enfermedad de tejido conectivo (vasculitis: granulomatosis necrotizante con poliangeitis, LES) ${ }^{(9)}$ (Figura 6).

\begin{tabular}{|l|}
\hline \multicolumn{1}{|c|}{ Dg diferencial nódulos cavitados } \\
\hline - Embolías sépticas (EBSA, CVC, marcapasos, \\
abuso drogas EV) \\
- Metástasis pulmonares \\
- Infecciones crónicas granulomatosas \\
(Hongos p/ej: Aspergiloma angioinvasiva, TBC) \\
- Linfoma (Especialmente en pacientes VIH) \\
- Nódulos reumatoídeos \\
- Enfermedad tejido conectivo \\
(Granulomatosis de Wegener, LES)
\end{tabular}

Figura 6. Diagnóstico diferencial de nódulos cavitados pulmonares.

La identificación de imágenes intraluminales en la arteria pulmonar no es un hallazgo esperable, ya que los infartos sépticos son casi invariablemente consecuencia de pequeños émbolos que se alojan en la vasculatura pulmonar distal ${ }^{(7,8)}$.

En cuanto a la evaluación de tromboflebitis de la VYI, tanto la TCMC con contraste como la US Doppler son muy sensibles en la detección de trombosis. Dentro de las etiologías de la trombosis, se describen: neoplasias (Síndrome paraneoplásico), trombofilias, abuso drogas endovenosas, cateterismo venoso central y otros procesos infecciosos-supurativos de cabeza y cuello (Figura 7) ${ }^{(13)}$.

Ante la sospecha de trombosis de VYI, la US Doppler cervical es el estudio de primera línea con una sensibilidad y especificidad de 92 y $98 \%$ respectivamente. La ausencia de flujo al Doppler color y la falta de compresibilidad de la vena representan hallazgos de alta sensibilidad, mientras que el signo mas específico es la visualización del trombo. Típicamente se observa una vena expandida, no compresible y ocupada por material en su interior (trombo), habitualmente ecogénico, lo que revela cierta temporalidad. Si el trombo es reciente por lo general se observa una vena expandida por material isoecogénico respecto del lumen (trombo blando). Cuando el trombo es oclusivo veremos ausencia completa de compresibilidad y flujo al doppler color, mientras que en el caso de un trombo parcialmente oclusivo podremos detectar flujo parcial y cierto grado de compresibilidad de la vena, hallazgos que pueden determinar un falso negativo para tomboflebitis en un operador no experimentado ${ }^{(14-16)}$ (Figura 8).

\begin{tabular}{|l|}
\hline \multicolumn{1}{|c|}{ Dg diferencial trombosis VYI } \\
\hline - Absceso retrofaríngeo \\
- CVC \\
- Drogas ev \\
- Neoplasias \\
\hline
\end{tabular}

Figura 7. Diagnóstico diferencial de trombosis vena yugular interna.

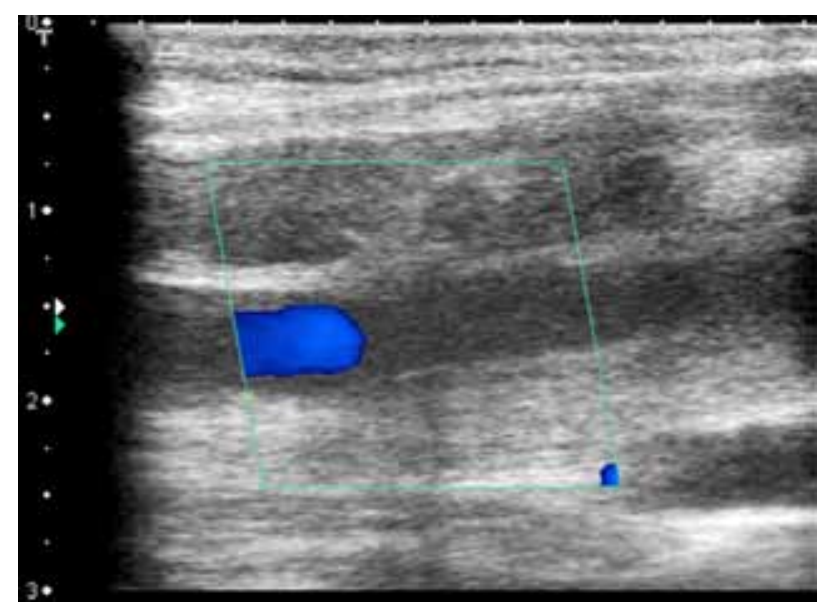

Figura 8. Caso 2, Eco Doppler color: Demuestra vena yugular interna derecha expandida con trombo isoecogénico, con ausencia de flujo.

La TCMD permite visualizar de forma completa la vena yugular interna, como también la posible extensión de la trombosis a las estructuras vasculares venosas del tórax. En fase aguda, veremos una vena expandida y ocupada por contenido con densidad de partes blandas que no se impregna con el contraste intravenoso, que representa el trombo endoluminal de localización central (Figuras 9 y 10). En fase crónica cuando existe recanalización, se aprecia un trombo mural excéntrico (organizado) y paredes engrosadas de la vena que realzan con el contraste intravenoso. 


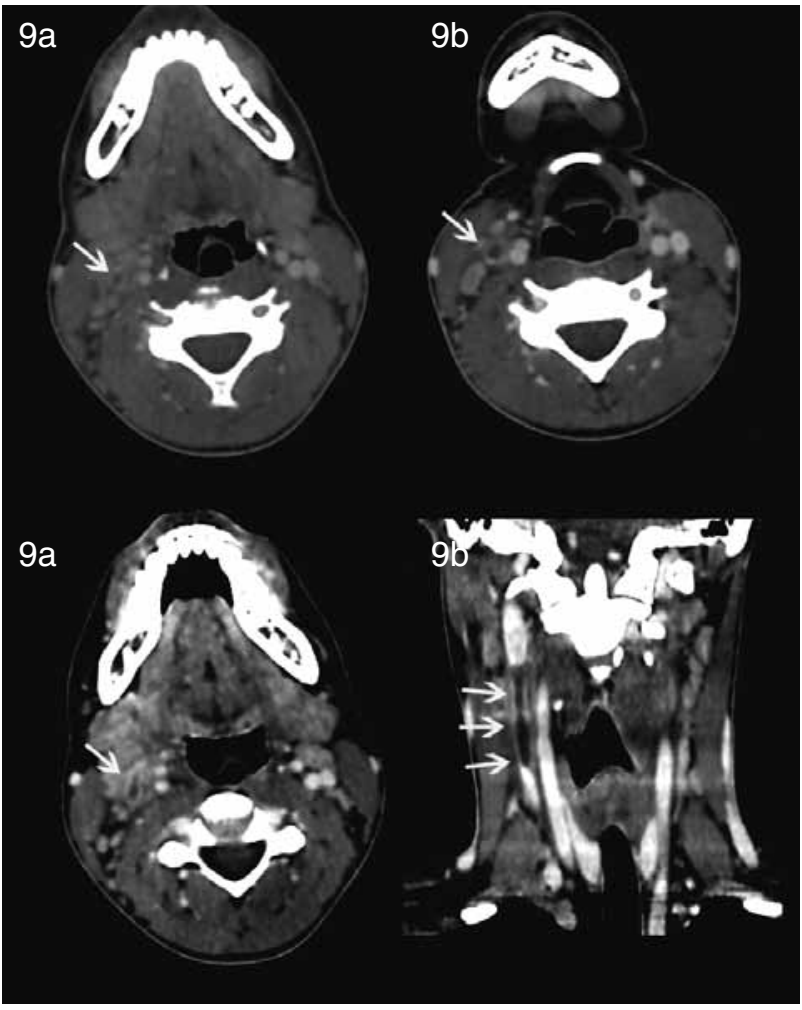

Figura 9. Caso 2, TC cuello con cte, cortes axiales y MPR coronal: En cortes axiales ( $a, b$ y $c)$ y corte coronal (d) se puede observar un defecto de llenado central de aproximadamente $4 \mathrm{~cm}$ de longitud de la vena yugular interna derecha, compatible con trombosis venosa (flechas).

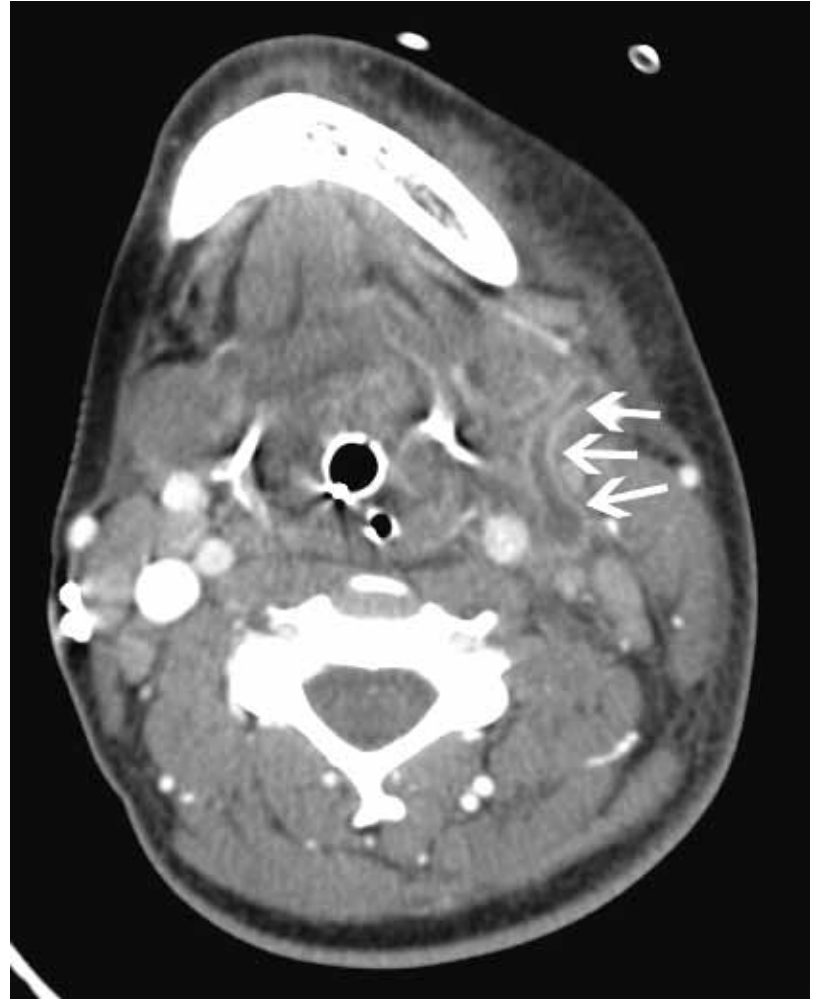

Figura 10. Caso 3, TC cuello con cte: Se evidencia aumento de volumen cervical izquierdo y en el lumen de la VYI izquierda existe imagen hipodensa compatible con trombo, las paredes venosas que captan contraste intravenoso (flechas).

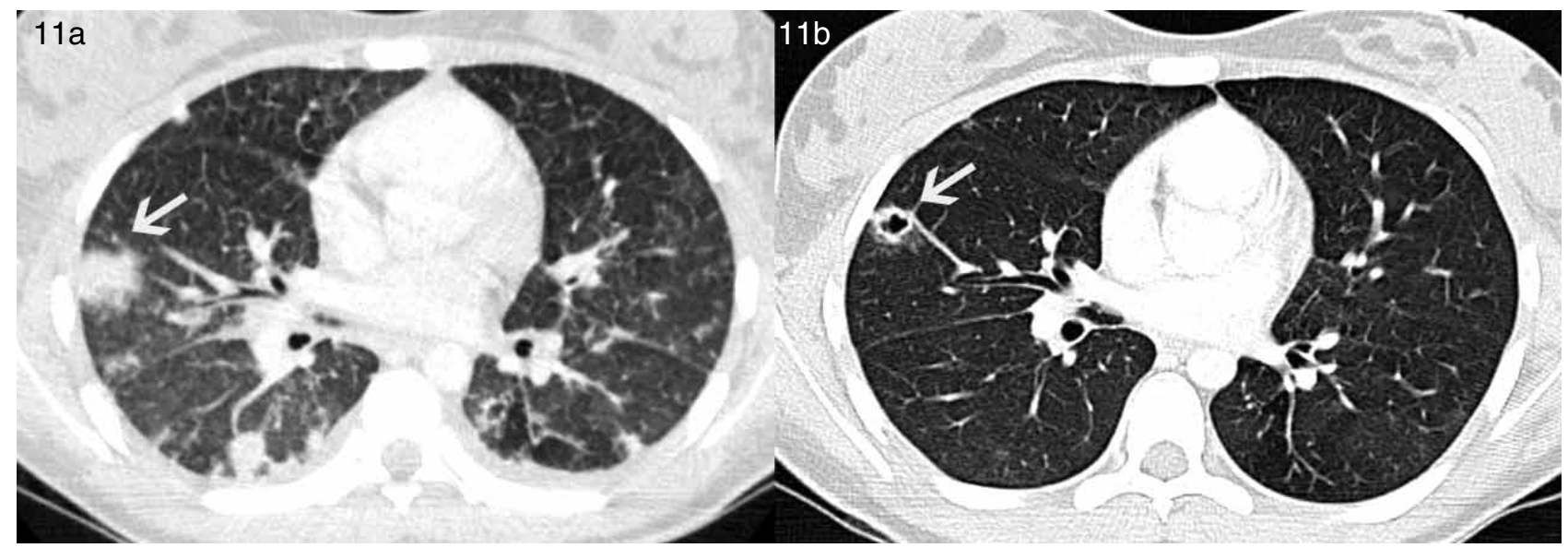

Figura 11. Caso 3, a) TC Tórax al ingreso demuestra opacidades nodulares de localización subpleurales, de predominio hacia las bases pulmonares, algo mayor a derecha (flechas). b) Control evolutivo a los 10 días evidencia cavitación de algunos nódulos, concordantes con embolías sépticas. Nótese favorable respuesta al tratamiento antibiótico con significativa disminución de las opacidades parenquimatosas.

\section{Discusión}

El síndrome de Lemierre es una entidad clínica de alta morbi-mortalidad de no ser diagnosticada y tratada en forma oportuna. Durante los años 60 y 70 , cuando la penicilina era el antibiótico más utilizado para tratar las infecciones faríngeas, este síndrome era conocido como "la enfermedad olvidada" por su escasa incidencia. Si bien sigue siendo una patología poco frecuente, hoy en día ha habido un aumento en su incidencia probablemente relacionada con la disminución del uso de antibióticos para tratar síntomas del tracto respiratorio alto, la disminución 
de tonsilectomías, el aumento de resistencia a los macrólidos y la mejora en los métodos de diagnóstico mediante imágenes y cultivos ${ }^{(1,17)}$.

El pronóstico de este síndrome es favorable de existir un diagnóstico y tratamiento oportunos. La mortalidad ha disminuido desde la serie inicial del Doctor Lemierre desde un $90 \%$ a una tasa de $4-18 \%{ }^{(18)}$.

Si bien el cuadro clínico del síndrome Lemierre es muy característico, actualmente los médicos no suelen estar familiarizados con esta patología ni con su forma de presentación, en parte debido a su baja incidencia y a que sus diagnósticos diferenciales son vastos. Cabe mencionar que la presencia de la triada clásica de este síndrome es infrecuente lo que dificulta el diagnóstico.

\section{Resumen}

El síndrome de Lemierre es un cuadro clínico poco frecuente pero potencialmente letal, de no existir un diagnóstico oportuno. Se debe dar especial énfasis en reconocer los hallazgos imagenológicos que permitirán plantear este síndrome, especialmente en el contexto de un paciente joven, inmunocompetente, con evolución tórpida de faringoamigdalitis que cursa con trombosis de VYI y manifestaciones de embolias sépticas a distancia.

\section{Bibliografía}

1. Aggarwal S, Nath A, Singh R, Keshri A. Lemierre's Syndrome presenting with neurological and pulmonary symptoms: Case report and review of the literature. Ann Indian Acad Neurol 2013; 16(2): 259-263.

2. Kristensen L, Prag J. Human necrobacillosis, with emphasis on Lemierre's syndrome. Clinical infectious diseases 2000; 31(2): 524-532.

3. Busko $M$, Triner $W$. Lemierre syndrome in a child with recent pharyngitis. Can J Emerg Med 2004; 6(4): 285-287.

4. Jankowich M, El-Sameed Y. A, Abu-Hijleh M. A 21-yearold man with fever and sore throat rapidly progressive to hemoptysis and respiratory failure. CHEST Journal 2007; 132(5): 1706-1709.

5. Dirks J, Bowie D. Sore throat progressing to embolic sepsis: a case of Lemierre's syndrome. Can Respir J 2010; 17(1): e20-22.

6. Chirinos JA, Lichtstein DM, Garcia J, Tamariz LJ. The evolution of Lemierre syndrome: report of 2 cases and review of the literature. Medicine 2002; 81(6): 458-465.

7. Hansell D, Armstrong P, Lynch D, McAdams HP. Infections of the lungs and pleura. Hansell D, Armstrong $P$, Lynch $D$, McAdams H.P. Imaging of diseases of the chest. 4th edition. Elsevier Mosby 2005; 183-276.

8. Hansell D, Armstrong P, Lynch D, McAdams HP. Pulmonary vascular diseases and pulmonary edema. Hansell $D$, Armstrong P, Lynch D, McAdams HP. Imaging of diseases of the chest 4th edition. Elsevier Mosby 2005; 361-428.

9. Nestor L, Muller C, Silva I. Embolia pulmonar nao trombótica e aneurisma de artéria pulmonar. Silva C, D'Ippolito G, José da Rocha A. Tórax. 1ed. Rio de Janeiro. Elsevier 2011; pp 191-198.

10. Tapia-Viñé M, González-García B, Bustos A, Cabello J. Manifestaciones radiológicas del Síndrome de Lemierre: a propósito de un caso. Radiología 2001; 43(2): 83-85.

11. Huang RM, Naidich DP, Lubat E, Schinella R, Garay SM, McCauley DI. Septic pulmonary emboli: CT-radiographic correlation. American Journal of Roentgenology 1989; 153(1): 41-45.

12. Dodd JD, Souza CA, Müller NL. "High-Resolution MDCT of Pulmonary Septic Embolism: Evaluation of the Feeding Vessel Sign." AJR 2006; 187(3): 623-29.

13. Mañas-García M. D, Marchán-Carranza E, Ceres-Alabau F, Rodríguez-Álvarez S. Trombosis de la vena yugular interna en dos pacientes en tratamiento con anticonceptivos orales. Med Clin (Barc) 2006; 127(5): 196-199.

14. Chin EE, Zimmerman PT, Grant G. "Sonographic Evaluation of Upper Extremity Deep Venous Thrombosis." Journal of Ultrasound in Medicine: Official Journal of the American Institute of Ultrasound in Medicine 2005; 24(6): 829-838; quiz 839-840.

15. Mueller D, V Lopez Rowe. "Internal Jugular Vein Thrombosis," March 19, 2014. http://emedicine.medscape.com/ article/461577-overview\#aw2aab6b2b1aa

16. Rumack C. Diagnostic Ultrasound. 4th Ed. Mosby; Enero 5, 2011.

17. Aljohaney A, McCarthy A. Lemierre's syndrome with paradoxical emboli. Internal medicine (Tokyo, Japan) 2009; 49(14): 1433-1436.

18. Syed MI, Baring D, Addidle M, Murray C, Adams C. Lemierre syndrome: Two cases and a review. The Laryngoscope 2007; 117(9): 1605-1610. 